\title{
COVID-19 Pneumonia Precipitating Acute Anterior Wall Myocardial Infarction With Large Left Ventricular Apical Thrombus
}

\author{
Sabu Johnª, b, Sudhanva Hegdea, Syed Hussain ${ }^{a}$, Inna Bukharovicha, Suzette Graham-Hilla, \\ Mary Mallappallila, Ronald Pedalino ${ }^{\mathrm{a}}$
}

\begin{abstract}
Initial reports suggest an increased thrombotic risk in coronavirus disease 2019 (COVID-19). We present a case of COVID-19 pneumonia that precipitated chest pain, an acute anterior wall ST-elevation myocardial infarction on the fifth day of hospitalization resulting in large left ventricular apical thrombus.
\end{abstract}

Keywords: Coronavirus disease 2019; ST-elevation myocardial infarction; Left ventricular apical thrombus

\section{Introduction}

Acute infections have been associated with a transient risk of venous thromboembolism (VTE). Coronavirus disease 2019 (COVID-19) is a new disease which is associated with high risk of thromboembolism. Preliminary data suggest that systemic anticoagulation in the setting of elevated D-dimer levels may reduce the thromboembolic risk [1]. A retrospective analysis suggests that attention should be paid to prophylaxis of VTE in the management of COVID-19 [2].

Data on non-myocarditis-related cardiovascular manifestations in COVID-19 are limited at present [3].

We present a case of rapid left ventricular (LV) thrombus formation in the setting of anterior ST-elevation myocardial infarction (STEMI) associated with COVID-19 pneumonia. A large apical thrombus was noted in two-dimensional echocardiogram within $24 \mathrm{~h}$ of onset of chest pain. The thrombus completely resolved with full anticoagulation in 6 weeks.

Manuscript submitted February 26, 2021, accepted March 12, 2021

Published online March 24, 2021

aDivision of Cardiovascular Disease, Department of Medicine, Kings County Hospital Center, Brooklyn, NY 11203, USA

${ }^{b}$ Corresponding Author: Sabu John, Division of Cardiovascular Disease, Department of Medicine, Kings County Hospital Center, Brooklyn, NY 11203, USA.Email: sabujohn67@aol.com

doi: https://doi.org/10.14740/jmc3687

\section{Case Report}

A 58-year-old man with no prior medical history presented with dry cough, shortness of breath, subjective fever, chills and fatigue for few days. He complained of increasing dyspnea on exertion with minimal physical activity. He was on no medications at home and a non-smoker. He had no significant family history and had no allergy.

On presentation, vital signs included a blood pressure (BP) of $137 / 91 \mathrm{~mm} \mathrm{Hg}$, heart rate (HR) of 95 beats per minute (bpm), temperature of $38.8^{\circ} \mathrm{C}$ (oral), respiratory rate (RR) of $19 / \mathrm{min}$ and oxygen saturation $\left(\mathrm{SPO}_{2}\right)$ on room air of $90-91 \%$ with desaturation to $85-88 \%$ on minimal exertion. Admission chest X-ray (Fig. 1) was read as bilateral patchy infiltrates consistent with COVID-19 pneumonia. Admission electrocardiogram (EKG) was normal. COVID-19 which was done by PCR (nasopharyngeal swab) on admission was positive. Initial D-dimer on admission was elevated to $629 \mathrm{ng} / \mathrm{mL}$ (normal 0 - $243 \mathrm{ng} / \mathrm{mL}$ ). Treatment was started with empiric antibiotics and oxygen. In addition, he was given guaifenesin for cough, acetaminophen for fever, enoxaparin $40 \mathrm{mg}$ subcutaneous daily for deep vein thrombosis (DVT) prophylaxis and pantoprazole for gastrointestinal (GI) prophylaxis.

From days 2 to 4 of admission, his respiratory status remained stable with pulse oximeter saturation being maintained at $96-98 \%$ with $4 \mathrm{~L} \mathrm{O}_{2}$ via nasal cannula (NC). On day 5 around 03:00, patient had chest pain which he described as blunt pain in the chest. At the time he was noted to desaturate to $88 \%$ on $3 \mathrm{~L}$ of oxygen via $\mathrm{NC}$ which was up titrated to 5 $\mathrm{L}$ of oxygen to maintain his $\mathrm{O}_{2}$ saturation at about $98 \%$. The rest of his vital signs showed temperature of $37.1^{\circ} \mathrm{C}$, HR of $78 \mathrm{bpm}$, RR of $18 / \mathrm{min}$ and $\mathrm{BP}$ of $133 / 98 \mathrm{~mm} \mathrm{Hg}$. A repeat chest X-ray (Fig. 2) was done which showed bilateral infiltrates. EKG done at the time of chest pain (Fig. 3) showed normal sinus rhythm without any ischemic changes and was similar to the admission EKG. EKG was repeated in an hour and did not show any significant changes. Patient had mild chest discomfort which resolved around afternoon with antacids. Initial troponin T was $0.01 \mathrm{ng} / \mathrm{mL}$ which was normal. The following day, patient complained of mild chest pain. Morning laboratory tests revealed elevated troponin $\mathrm{T}$ of $5.660 \mathrm{ng} /$ $\mathrm{mL}$ (normal range $\leq 0.010 \mathrm{ng} / \mathrm{mL}$ ). Repeat EKG (Fig. 4) with return of chest pain revealed an anterolateral wall myocardial 

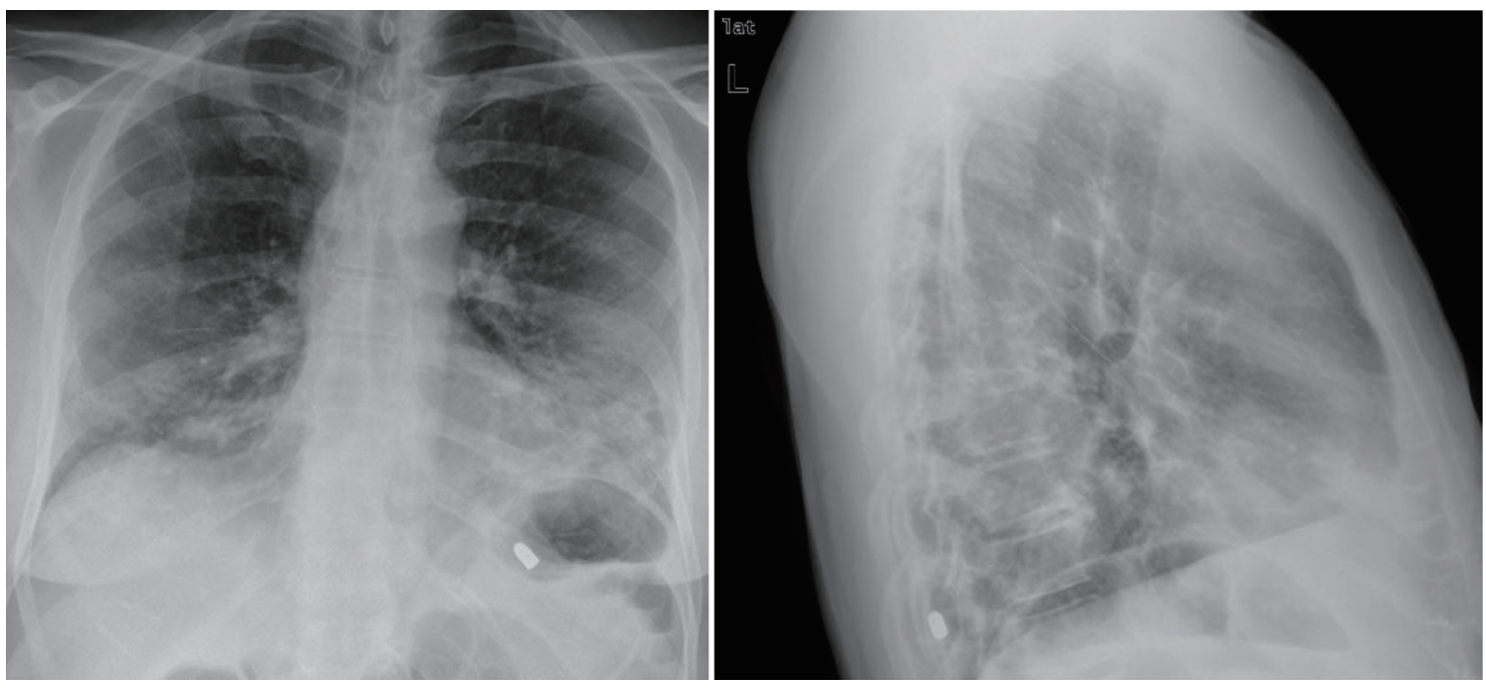

Figure 1. Admission chest $\mathrm{X}$-ray showing bilateral patchy infiltrates.

infarction.

An echocardiogram (Fig. 5) done at the same time showed antero-septal and apical akinesia with large apical thrombus and an ejection fraction of $25 \%$. There was a concern for using thrombolytic therapy due to possible disintegration of the thrombus which could lead to embolic complications. Cardiac catheterization and transfer for primary percutaneous coronary intervention (PCI) was deferred due to significant COVID-19 pneumonia with hypoxemia along with a large LV thrombus. Patient was also chest pain free at that time. Treatment was started immediately with enoxaparin $1 \mathrm{mg} / \mathrm{kg}$ subcutaneously twice daily for full anticoagulation. A loading dose of clopidogrel $300 \mathrm{mg}$ was given orally along with aspirin. Sacubitrilvalsartan 24 - $26 \mathrm{mg}$ twice daily orally and metoprolol succinate extended release $50 \mathrm{mg}$ daily orally was also started for systolic heart failure. Patient's symptoms resolved and he was discharged home on oral anticoagulant apixaban $5 \mathrm{mg}$ twice daily, clopidogrel $75 \mathrm{mg}$ daily orally, high-dose statin and heart failure medications. A repeat echocardiogram done

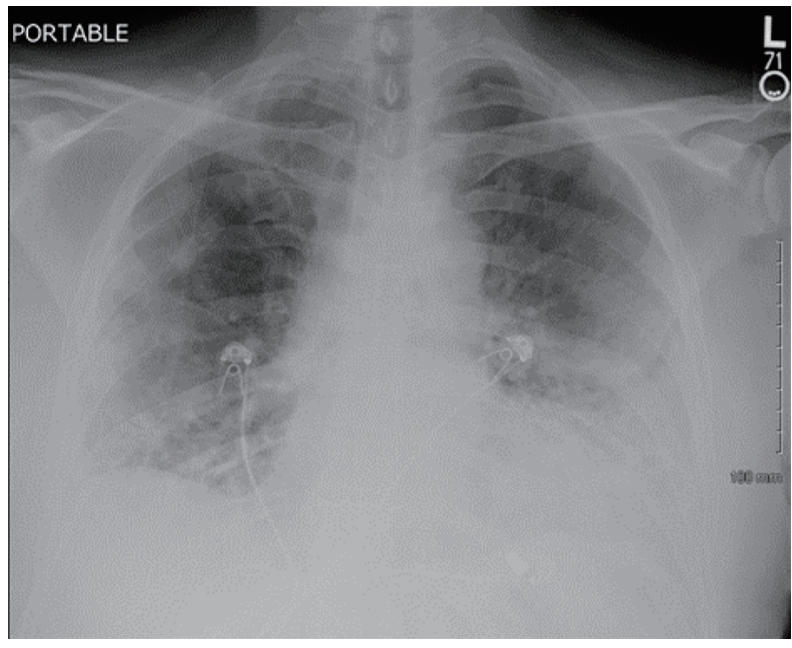

Figure 2. Repeat chest X-ray showing bilateral infiltrates. as outpatient in 6 weeks showed complete resolution of apical thrombus and an improvement in ejection fraction to $35 \%$. Another echocardiogram done 5 months later showed normal ejection fraction and no LV thrombus. Apixaban was discontinued and patient was advised to continue clopidogrel $75 \mathrm{mg}$ orally daily along with aspirin $81 \mathrm{mg}$ daily. Echocardiogram (Fig. 5) showed antero-septal and apical akinesis with large LV thrombus.

\section{Discussion}

A combination of blood stasis, endothelial injury and hypercoagulability contributes to in vivo thrombus formation [4]. All of these requirements were present in the above case. In the setting of a myocardial infarction, LV akinesia of the anterior wall, apex and septum can result in stasis of blood and may also have contributed to the intraventricular thrombus formation. Inflammation in the setting of the COVID-19 infection may have contributed to a hypercoagulable state, resulting in the rapid formation of a large LV thrombus in the setting of an acute STEMI. Large thrombus formation is highly unusual in the setting of acute myocardial infarction alone within $24 \mathrm{~h}$ of symptom onset.

Acute anterior wall STEMI involving large area of myocardium can cause intraventricular thrombus, usually at the apex, within 1 or 2 weeks of presentation [5]. The median time to thrombus formation is 5 - 6 days, but has been reported to occur as early as $24 \mathrm{~h}$ but not a large thrombus of this size [6]. The incidence of intracardiac thrombus formation in the prerevascularization era was noted to be as high as $40 \%$, which required systemic anticoagulation with warfarin for 3 months [7]. The incidence of thrombus formation after an acute STEMI has significantly decreased since the start of primary PCI therapy [8]. Subsequently, in patients treated with primary PCI for left anterior descending artery occlusion, the incidence of thrombus formation was noted to be about $4 \%$. There was also a reduction in post STEMI intracardiac thrombus formation 


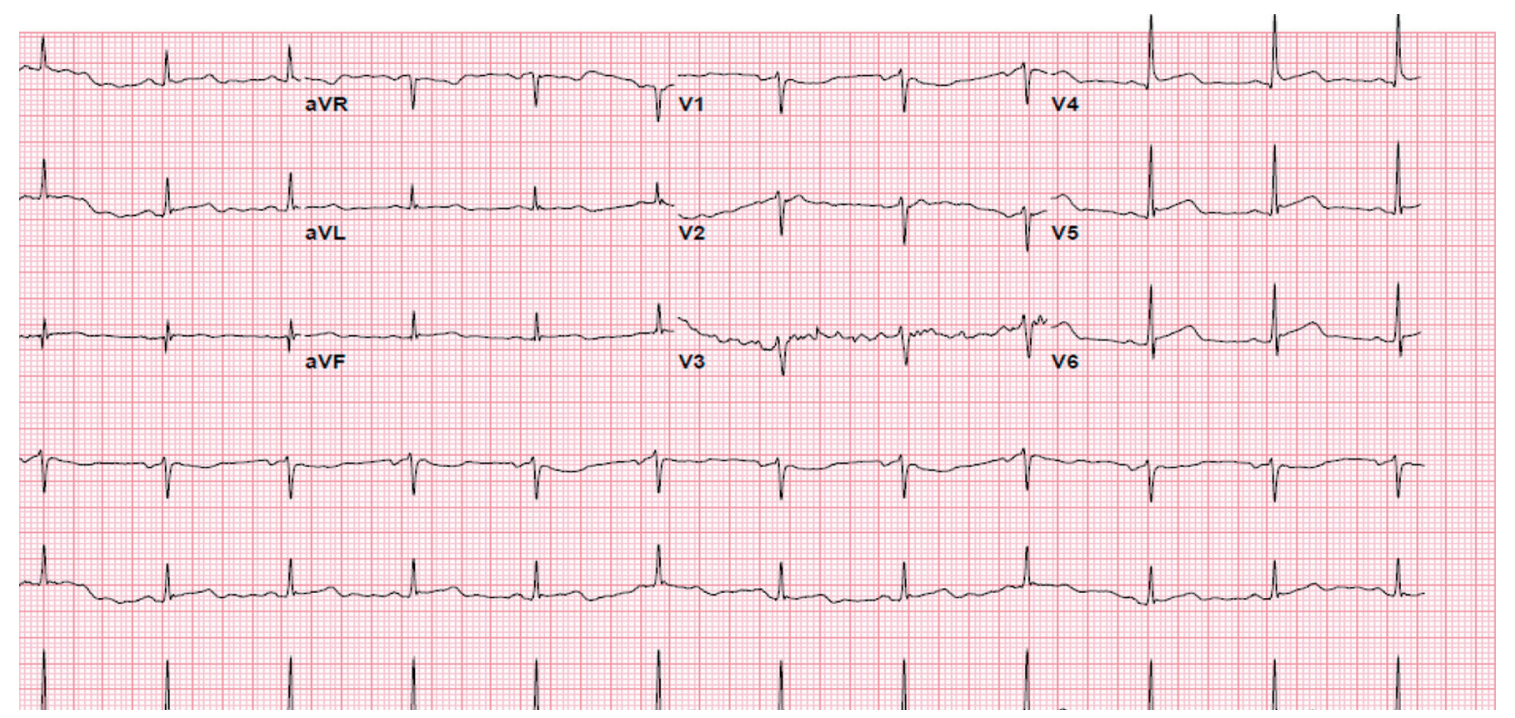

Figure 3. Electrocardiogram at the time of chest pain.

with fibrinolysis [9].

Our patient presented to emergency room with fever, cough, dyspnea and hypoxemia during COVID-19 pandemic peak in New York. Patient was tested positive for COVID-19. He had no significant past medical history and was otherwise healthy. Treatment was started with empiric antibiotics and oxygen via NC. He was comfortable and ambulating. Inflammatory markers were significantly elevated.

Admission EKG was normal. Initial EKG at the time of the chest pain was normal with no ischemic changes. Initial troponin was normal. The following day, given recurrence of vague chest discomfort, a repeat troponin was done which was significantly elevated. A repeat EKG on the following day showed anterior ST elevation. An echocardiogram was performed to decide therapeutic options. It showed anterior, anteroseptal and apical akinesia with large thrombus filling the apex to almost one-third of the LV cavity. The decision was made for full anticoagulation and plavix.

There was a concern for using thrombolytic therapy due to possible disintegration of the thrombus which could lead to embolic complications. The rapid formation of LV apical thrombus suggests hypercoagulable state. Given the clinical stability of the patient and hypercoagulable state, medical therapy was deemed the best option compared to primary PCI. Patient was discharged home on day 14 since admission with full anticoagulation, aspirin and clopidogrel. There was no need for home oxygen on discharge and the LV thrombus resolved with full anticoagulation alone in 6 weeks.

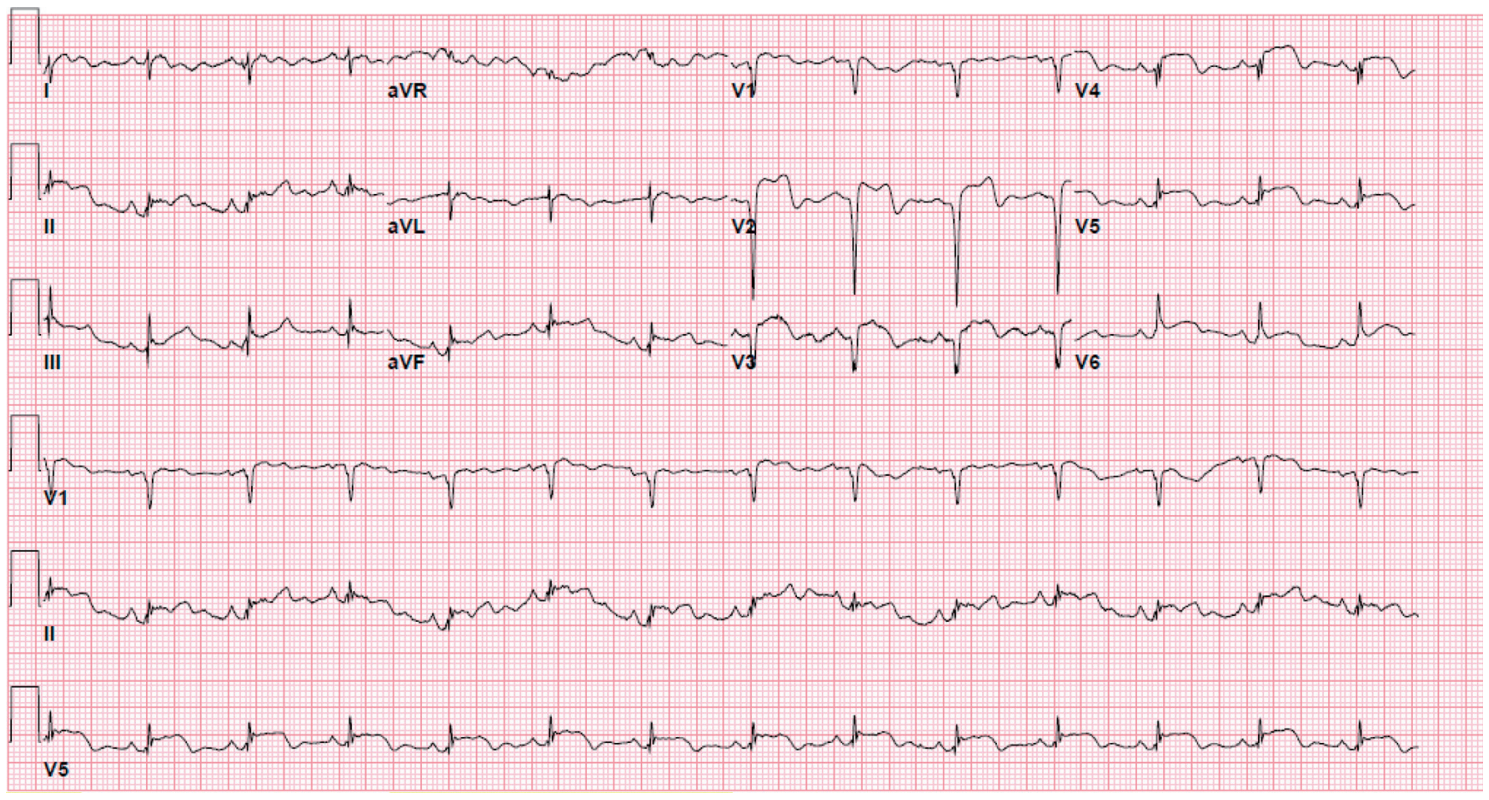

Figure 4. Repeat electrocardiogram the following day showing anterolateral ST elevation. 

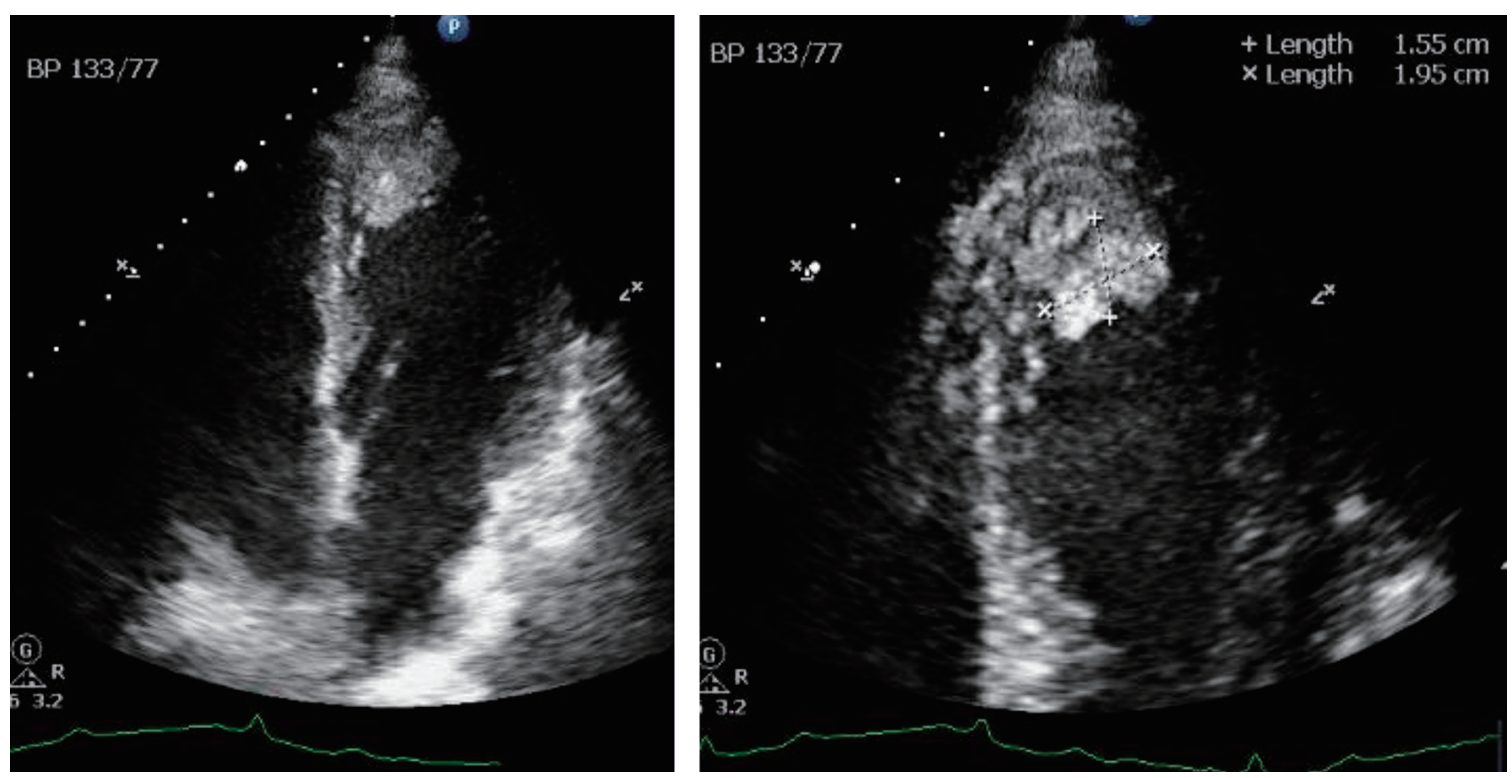

Figure 5. Apical view of echocardiogram showing large apical thrombus.

There is growing evidence now about COVID-19 infection, causing hypercoagulable state. Full anticoagulation may be warranted, in certain subset of patients, especially when the D-dimer is significantly elevated with no evidence of bleeding and without any contraindications.

\section{Acknowledgments}

None to declare.

\section{Financial Disclosure}

None to declare.

\section{Conflict of Interest}

None to declare.

\section{Informed Consent}

Verbal consent was obtained over the phone, documented and witnessed. Patient is doing fine and agreed for publication.

\section{Author Contributions}

All authors reviewed the echos, EKGs and chart together. Sabu John wrote the manuscript. Sudhanva Hegde, Syed Hussain, Inna Bukharovich, Suzette Graham-Hill, Mary Mallappallil and Ronald Pedalino edited the manuscript. All authors agreed the final version.

\section{Data Availability}

The authors declare that data supporting the findings of this study are available within the article.

\section{References}

1. Tang N, Bai H, Chen X, Gong J, Li D, Sun Z. Anticoagulant treatment is associated with decreased mortality in severe coronavirus disease 2019 patients with coagulopathy. J Thromb Haemost. 2020;18(5):1094-1099.

2. Wang T, Chen R, Liu C, Liang W, Guan W, Tang R, Tang $\mathrm{C}$, et al. Attention should be paid to venous thromboembolism prophylaxis in the management of COVID-19. Lancet Haematol. 2020;7(5):e362-e363.

3. Bansal M. Cardiovascular disease and COVID-19. Diabetes Metab Syndr. 2020;14(3):247-250.

4. Delewi R, Zijlstra F, Piek JJ. Left ventricular thrombus formation after acute myocardial infarction. Heart. 2012;98(23):1743-1749.

5. Nihoyannopoulos P, Smith GC, Maseri A, Foale RA. The natural history of left ventricular thrombus in myocardial infarction: a rationale in support of masterly inactivity. J Am Coll Cardiol. 1989;14(4):903-911.

6. Greaves SC, Zhi G, Lee RT, Solomon SD, MacFadyen J, Rapaport E, Menapace FJ, et al. Incidence and natural history of left ventricular thrombus following anterior wall acute myocardial infarction. Am J Cardiol. 1997;80(4):442-448.

7. Asinger RW, Mikell FL, Elsperger J, Hodges M. Incidence of left-ventricular thrombosis after acute transmural myocardial infarction. Serial evaluation by two-dimensional echocardiography. N Engl J Med. 1981;305(6):297302. 
8. Rehan A, Kanwar M, Rosman H, Ahmed S, Ali A, Gardin J, Cohen G. Incidence of post myocardial infarction left ventricular thrombus formation in the era of primary percutaneous intervention and glycoprotein IIb/IIIa inhibitors. A prospective observational study. Cardiovasc
Ultrasound. 2006;4:20.

9. Vecchio C, Chiarella F, Lupi G, Bellotti P, Domenicucci $\mathrm{S}$. Left ventricular thrombus in anterior acute myocardial infarction after thrombolysis. A GISSI-2 connected study. Circulation. 1991;84(2):512-519. 